\title{
On computing nullspace bases - a fault detection perspective
}

\author{
Andras Varga \\ German Aerospace Center, DLR-Oberpfaffenhofen \\ Institute of Robotics and Mechatronics \\ D-82234 Wessling, Germany \\ Email: andras.varga@dlr.de
}

\begin{abstract}
We discuss computationally efficient and numerically reliable algorithms to compute minimal proper nullspace bases of a rational or polynomial matrix. The underlying main computational tool is the orthogonal reduction to a Kronecker-like form of the system matrix of an equivalent descriptor system realization. A new algorithm is proposed to compute a simple minimal proper nullspace basis, starting from a non-simple one. Minimal dynamic cover based computational techniques are used for this purpose. The discussed methods allow a high flexibility in addressing in a numerically sound way several applications in fault detection.
\end{abstract}

Keywords: Fault detection, nullspace method, rational bases, numerical methods.

\section{INTRODUCTION}

The nullspace method to design residual generators for fault detection has been formally introduced by Frisk and Nyberg (2001), where a polynomial basis based approach was used. This approach has been later extended to rational bases by Varga (2003a, 2007c). The main advantage of this approach is that the least order design aspect is naturally present in the formulation of the method. In a recent survey (Varga, 2007a), it was shown that the nullspace method also provides a unifying design paradigm for most of existing approaches, which can be interpreted as special cases of this method. The main computation in nullspace methods is to determine a rational (polynomial or proper) basis for the nullspace of a certain rational matrix.

Consider a $p \times m$ rational matrix $G(\lambda)$, where the indeterminate $\lambda$ is a complex variable. If we interpret $G(\lambda)$ as the transfer-function matrix (TFM) of a linear time-invariant system, then according to the system type, $\lambda$ is the $s$ variable in the Laplace transform in the case of a continuoustime system or the $z$ variable in the $Z$-transform in the case of a discrete-time system. This interpretation of $\lambda$ is relevant when system stability aspects are considered.

We assume that $G(\lambda)$ is a rational or polynomial matrix with normal rank $r$. In view of fault detection applications, we consider the computation of a $(p-r) \times p$ rational basis matrix $N_{l}(\lambda)$ of the left nullspace of $G(\lambda)$ such that

$$
N_{l}(\lambda) G(\lambda)=0 .
$$

Of special importance are minimal bases having the least achievable McMillan degree. Moreover, depending on the underlying application, further desirable properties may be achieved, as for example, determining $N_{l}(\lambda)$ as a polynomial matrix or as a proper rational matrix with specified poles.
In most of practical applications, the rational matrix $G(\lambda)$ is implicitly given via a descriptor system representation

$$
G(\lambda):=\left[\begin{array}{c|c}
A-\lambda E & B \\
\hline C & D
\end{array}\right]
$$

which satisfies

$$
G(\lambda)=C(\lambda E-A)^{-1} B+D
$$

For a proper $G(\lambda)$ we can always choose $E=I$. In our succinct discussion of computational methods which follows, we assume that such a realization is the primary data for nullspace computations.

The rigorous study of polynomial bases started with the theoretical works of Forney (1975), and followed by initial algorithmic developments of Kailath (1980). For the computation of a minimal polynomial bases of a polynomial matrix $G(\lambda)$ there are many algorithms, see (Antoniou et al., 2005) and the literature cited therein. Two main classes of methods are the resultant methods, which determine the solution by solving directly polynomial equations involving appropriate resultant matrices (Antoniou et al., 2005), and pencil methods, which rely on matrix pencil reduction algorithms (Beelen, 1987). While resultant methods can be considered a real alternative to the unreliable polynomial manipulation based methods proposed in (Kailath, 1980), their application requires, as a supplementary step, bringing the system model into a polynomial representation. This involve factoring $G(\lambda)$ as $G(\lambda)=N(\lambda) M^{-1}(\lambda)$, where $N(\lambda)$ and $M(\lambda)$ are polynomial matrices, and applying the method to $N(\lambda)$. The converse operation (e.g., proper rational factoring of a polynomial matrix) is also necessary, if the desired basis is a proper rational basis. Such computational detours are generally considered highly unreliable for large scale systems, which usually arise in a state-space form.

The pencil methods rely on a state space or descriptor system realization of $G(\lambda)$ of the form (1), and are appli- 
cable to both polynomial and rational matrices. The main computational tool is the reduction of a matrix pencil to a Kronecker-like form using orthogonal transformations. The left Kronecker structure provides the complete information to compute a polynomial basis via straightforward polynomial matrix manipulations Beelen (1987).

For many applications, proper rational bases are required. Such bases can be immediately obtained from polynomial bases. However, to avoid potentially unstable polynomial manipulations, it is of interest to compute proper rational bases directly, without the unnecessary detour of determining first polynomial bases. The theory of proper rational bases has been developed by Vardulakis and Karcanias (1984), where the main concepts have been defined. Of special importance are proper bases which are simple (see the exact definition in the next section), representing a direct generalization of minimal polynomial bases. A first reliable numerical method to compute proper rational bases has been proposed by Varga (2003a). This method belongs to the class of pencil methods and its main advantage is that a minimal proper rational basis can be computed by using exclusively orthogonal transformations. Note however, that the resulting basis is generally not simple.

In this paper we focus on methods to compute rational nullspace basis which, in our opinion, are best suited for the specific needs of solving efficiently the fault detection problem with least order residual generators. We propose a new algorithm to compute simple minimal proper rational bases which can be seen as an extension of that proposed in (Varga, 2003a). The new algorithm determines a simple basis starting from a non-simple one. Minimal dynamic covers techniques are used for this purpose. The proposed algorithm allows to also compute linear combinations of basis vectors which immediately lead to candidate solutions of the fault detection problem with least order detector. New formulas for detector design are developed which allow to check the solvability conditions practically without extra computations.

\section{NULLSPACE BASES}

Since polynomial bases represent an important tool in defining the corresponding concepts for the more general rational bases, we will recall shortly some of the main results of Forney (1975). Assume that $N_{l}(\lambda)$ is a polynomial basis of the left nullspace of $G(\lambda)$. Let denote by $n_{i}$, the $i$-th index (or degree), representing the greatest degree of the $i$-th row of $N_{l}(\lambda)$. Then, the order of $N_{l}(\lambda)$ is defined as $n_{d}=\sum_{i=1}^{p-r} n_{i}$, (i.e., the sum of row degrees). A minimal basis is one which has least order among all polynomial bases. The indices of a minimal basis are called minimal indices. The order of a minimal polynomial basis $N_{l}(\lambda)$ is equal to the McMillan degree of $N_{l}(\lambda)$.

Some properties of a minimal bases are summarized below (Forney, 1975; Kailath, 1980):

Theorem 1. Let $N_{l}(\lambda)$ be a minimal polynomial basis with row indices $n_{i}, i=1, \ldots, p-r$. Then the following holds:

(1) The row indices are unique up to permutations (i.e., if $\widetilde{N}_{l}(\lambda)$ is another minimal basis, then $N_{l}(\lambda)$ and $\tilde{N}_{l}(\lambda)$ have the same minimal indices).
(2) The minimal indices are the left Kronecker indices of $G(\lambda)$.

(3) $N_{l}(\lambda)$ is irreducible, i.e., has full row rank for all $\lambda \in \mathbb{C}$ $\left(N_{l}(\lambda)\right.$ has no finite or infinite zeros).

(4) $N_{l}(\lambda)$ is row reduced, i.e., the leading row coefficient matrix (formed from the coefficients of the highest row degrees) has full row rank.

If $M(\lambda)$ is a non-singular rational matrix, then $\tilde{N}_{l}(\lambda):=$ $M(\lambda) N_{l}(\lambda)$ is also a nullspace basis. Frequently the matrices $M(\lambda)$ originate from appropriate left coprime factorizations of an original basis $N_{l}(\lambda)$ in the form

$$
N_{l}(\lambda)=M(\lambda)^{-1} \tilde{N}_{l}(\lambda),
$$

where the factors $M(\lambda)$ and $\widetilde{N}_{l}(\lambda)$ can be choosen to satisfy special requirements (e.g., have only poles in a certain "good" region of the complex plane).

The main advantage of minimal polynomial bases is the possibility to easily build proper minimal rational bases. These are proper rational bases having the least McMillan degree of $n_{d}$. A proper rational basis with arbitrary poles can be simply constructed by taking

$$
M(\lambda)=\operatorname{diag}\left(\frac{1}{d_{1}(\lambda)}, \cdots, \frac{1}{d_{p-r}(\lambda)}\right),
$$

where $d_{i}(\lambda)$ is a polynomial of degree $n_{i}$, and forming $\widetilde{N}_{l}(\lambda):=M(\lambda) N_{l}(\lambda)$. The resulting basis $\widetilde{N}_{l}(\lambda)$ has the additional property that the order of any minimal state space realization of $\widetilde{N}_{l}(\lambda)$ is equal to the sum of orders of the minimal state space realizations of the rows of $\widetilde{N}_{l}(\lambda)$. Furthermore, $D_{l}:=\lim _{\lambda \rightarrow \infty} \widetilde{N}_{l}(\lambda)$ has full row rank. Such a proper basis is termed simple in (Vardulakis and Karcanias, 1984) and is the natural counterpart of minimal polynomial basis introduced by Forney (1975).

Simple bases are useful when solving fault detection problems with least order detectors. For example, building linear combinations of basis vectors to obtain least order candidate detectors is very straightforward. Moreover, left coprime factorizations of the form (2) can be built with diagonal $M(\lambda)$, thus without altering the simple structure of the basis. In the next section we address the computation of a simple basis starting from a special proper rational basis computed using a numerically stable pencil method.

\section{COMPUTATION OF MINIMAL PROPER BASES}

For the computation of a rational nullspace basis $N_{l}(\lambda)$ a pencil method based on a state space representation of $G(\lambda)$ in the form (1) has been proposed in Varga (2003a). In this section we review this algorithm and give some of the properties of the resulting basis. Although minimal, it appears that the resulting basis is not simple. An approach to obtain simple bases is presented in the next section.

The computational method described in Varga (2003a) exploits the simple fact that $N_{l}(\lambda)$ is a left nullspace basis of $G(\lambda)$ iff $\left[M_{l}(\lambda) N_{l}(\lambda)\right]$ is a left nullspace basis of the system matrix

$$
S(\lambda)=\left[\begin{array}{cc}
A-\lambda E & B \\
C & D
\end{array}\right] .
$$


Thus, to compute $N_{l}(\lambda)$ we can determine equivalently a left nullspace basis $Y_{l}(\lambda)$ for $S(\lambda)$ and then $N_{l}(\lambda)$ simply results as

$$
N_{l}(\lambda)=Y_{l}(\lambda)\left[\begin{array}{c}
0 \\
I_{p}
\end{array}\right]
$$

$N_{l}(\lambda)$ can be computed by employing linear pencil reduction algorithms based on orthogonal transformations. The resulting nullspace is obtained in a descriptor system representation which can be immediately used in applications. In what follows we give some details of this approach.

Let $Q$ and $Z$ be orthogonal matrices (for instance, determined by using the algorithms of (Beelen, 1987; Varga, 1996) such that the transformed pencil $\widetilde{S}(\lambda):=Q S(\lambda) Z$ is in the Kronecker-like staircase form

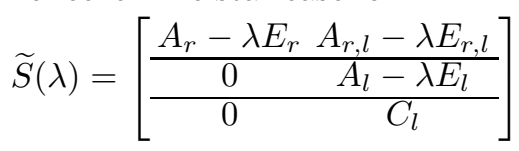

where the descriptor pair $\left(A_{l}-\lambda E_{l}, C_{l}\right)$ is observable, $E_{l}$ is non-singular, and $A_{r}-\lambda E_{r}$ has full row rank excepting possibly a finite set of values of $\lambda$ (i.e, the invariant zeros of $S(\lambda)$ ). It follows that we can choose the nullspace $\widetilde{Y}_{l}(\lambda)$ of $\widetilde{S}(\lambda)$ in the form

$$
\tilde{Y}_{l}(\lambda)=\left[0\left|C_{l}\left(\lambda E_{l}-A_{l}\right)^{-1}\right| I\right] .
$$

Then the nullspace of $G(\lambda)$ is

$$
N_{l}(\lambda)=\widetilde{Y}_{l}(\lambda) Q\left[\begin{array}{c}
0 \\
I_{p}
\end{array}\right]
$$

and if we partition

$$
Q\left[\begin{array}{c}
0 \\
I_{p}
\end{array}\right]=\left[\begin{array}{c}
B_{r, l} \\
B_{l} \\
D_{l}
\end{array}\right]
$$

in accordance with the column partition of $\widetilde{Y}_{l}(\lambda)$, we obtain

$$
N_{l}(\lambda)=C_{l}\left(\lambda E_{l}-A_{l}\right)^{-1} B_{l}+D_{l}:=\left[\begin{array}{c|c}
A_{l}-\lambda E_{l} & B_{l} \\
\hline C_{l} & D_{l}
\end{array}\right]
$$

which is a descriptor system representation for $N_{l}(\lambda)$. Note that, to obtain this nullspace basis, we performed exclusively orthogonal transformations on the system matrices. We can prove that all computed matrices are exact for a slightly perturbed original system. It follows that the algorithm to compute the nullspace basis is numerically backward stable.

The full column rank subpencil $\left[\begin{array}{c}A_{l}-\lambda E_{l} \\ C_{l}\end{array}\right]$ defines the left Kronecker structure of $G(\lambda)$. It is possible to obtain this subpencil in an observability staircase form

$\left[\begin{array}{c|c|c|c}A_{\ell, \ell+1} & A_{\ell, \ell}-\lambda E_{\ell, \ell} & \cdots & A_{\ell, 1}-\lambda E_{\ell, 1} \\ \hline & A_{\ell-1, \ell} & \ddots & \vdots \\ \hline & & \ddots & A_{1,1}-\lambda E_{1,1} \\ \hline & & & A_{0,1}\end{array}\right]$

where $A_{i, i+1} \in \mathbb{R}^{\mu_{i} \times \mu_{i+1}}$, with $\mu_{\ell+1}=0$, are full column rank upper triangular matrices, for $i=0, \ldots, \ell$. Note that this form is automatically obtained by using the pencil reduction algorithms described in (Beelen, 1987) or (Varga, 1996). The left (or row) Kronecker indices result as follows: there are $\mu_{i-1}-\mu_{i}$ Kronecker blocks of size $i \times(i-1)$, for $i=1, \ldots, \ell+1$. The row dimension of $N_{l}(\lambda)$ (i.e., the number of linearly independent basis vectors) is given by the total number of Kronecker indices, thus $\sum_{i=1}^{\ell+1}\left(\mu_{i-1}-\mu_{i}\right)=\mu_{0}$. Applying standard linear algebra results, it follows that $\mu_{0}:=p-r$.

We give now some properties of the computed rational basis (see (Varga, 2007a) for proofs).

Theorem 2. If the realization (1) of $G(\lambda)$ is minimal, then the rational matrix $N_{l}(\lambda)$ defined in (6) is a minimal proper rational basis of the left nullspace of $G(\lambda)$.

This result shows that the computed rational basis above has actually the least possible McMillan degree. However, in general, the computed minimal proper basis is not simple. Additionally the following important result holds: Proposition 3. If the realization (1) of $G(\lambda)$ is minimal, then the realization of $N_{l}(\lambda)$ defined in (6) is maximally controllable.

This means that for any output injection matrix $K$, the pair $\left(A_{l}+K C_{l}-\lambda E_{l}, B_{l}+K D_{l}\right)$ remains controllable.

\section{COMPUTATION OF SIMPLE BASES}

The most obvious approach to determine a simple minimal proper rational basis has been sketched in Section 2 and consists in computing first a minimal polynomial basis $N_{l}(\lambda)$ and then determining the rational basis as $\tilde{N}_{l}(\lambda):=$ $M(\lambda) N_{l}(\lambda)$, where $M(\lambda)$ has the form (3).

We discuss shortly the method to compute a polynomial basis proposed in Beelen (1987). This method determines first a minimal polynomial basis $V(\lambda)$ for the left nullspace of the sub-pencil $\left[\begin{array}{c}A_{l}-\lambda E_{l} \\ C_{l}\end{array}\right]$ in (4). This computation can be done by fully exploiting the staircase structure of this pencil (7) and details for a dual algorithm (for right basis) are presented in Beelen (1987). The degrees of the resulting left basis vectors are equal to the left Kronecker indices, and this information can be simply read out from the staircase structure. As already mentioned, there are $p-r$ basis vectors, of which there are $\mu_{i-1}-\mu_{i}$ vectors of degree $(i-1)$.

The minimal polynomial nullspace basis of $G(\lambda)$ results as

$$
N_{l}(\lambda)=V(\lambda) Q\left[\begin{array}{c}
0 \\
I_{p}
\end{array}\right]
$$

Note that $V(\lambda)$ and $N_{l}(\lambda)$ have the same row degrees. Furthermore, it is shown in Beelen (1987) that the resulting $N_{l}(\lambda)$ is row reduced.

The approach to compute a simple proper minimal basis has been sketched in Section 2 and additionally involves to determine $M(\lambda)$ of the form (3), where $d_{i}(\lambda)$ is an arbitrary polynomial of degree $n_{i}$. The resulting simple proper minimal basis is $\widetilde{N}_{l}(\lambda):=M(\lambda) N_{l}(\lambda)$. A statespace realization of the resulting basis $\widetilde{N}_{l}(\lambda)$ can be simply built by inspection, exploiting the simple structure 
property. This realization is obtained by simply stacking $p-r$ minimal realizations of orders $n_{i}, i=1, \ldots, p-r$ of each row of $\widetilde{N}_{l}(\lambda)$. The resulting state matrix has a block diagonal structure. Although simple, this approach is not best suited for fault detection applications for reasons mentioned in the Conclusions.

We propose an alternative to this method which is based on minimum cover techniques and, as will be shown later, is directly related to determining least order detectors. Consider the proper rational nullspace (6) and denote with $c_{l, i}$ and $d_{l, i}$ the $i$-th rows of matrices $C_{l}$ and $D_{l}$, respectively.

Theorem 4. For each $i=1, \ldots, p-r$, let $K_{i}$ be an output injection matrix such that

$$
v_{i}(\lambda):=c_{l, i}\left(\lambda E_{l}-A_{l}-K_{i} C_{l}\right)^{-1}\left(B_{l}+K_{i} D_{l}\right)+d_{l, i}
$$

has the least possible McMillan degree. Then, $V(\lambda)$ formed from the $p-r$ rows $v_{i}(\lambda)$ is a simple proper minimal rational basis.

Proof. The achieved McMillan degrees of each $v_{i}(\lambda)$ is the corresponding minimal index $n_{i}$, representing, in a dual setting, the dimension of the least order controllability subspace of the pair $\left(A_{l}^{T}-\lambda E_{l}^{T}, C_{l}^{T}\right)$ covering $c_{l, i}^{T}$. This result follows from (Warren and Eckberg, 1975, Theorem 1). Furthermore, the computed set of basis vectors $\left\{v_{1}(\lambda), \ldots, v_{p-r}(\lambda)\right\}$ is linearly independent.

The poles of the nullspace basis can be arbitrarily placed by performing left coprime rational factorizations

$$
v_{i}(\lambda)=d_{i}(\lambda)^{-1} \widetilde{v}_{i}(\lambda)
$$

The basis $\tilde{V}(\lambda):=\left[\widetilde{v}_{1}^{T}(\lambda), \ldots, \widetilde{v}_{p-r}^{T}(\lambda)\right]^{T}$ obtained in this way, can have arbitrarily assigned dynamics.

Remark. The importance of simple bases for solving fault detection problems is the following property. For a given row index $i$, let $h$ be a $(p-r)$-dimensional row vector having only the last $i$ components non-zero. Then, a linear combination of basis vectors not exceeding degree $n_{i}$ can be generated as

$$
v(\lambda):=h C_{l}\left(\lambda E_{l}-A_{l}-K C_{l}\right)^{-1}\left(B_{l}+K D_{l}\right)+h D_{l}
$$

where $K$ is an output injection matrix such that $v(\lambda)$ has the least possible order (at most $n_{i}$ ). This matrix can be determined, using the minimal dynamic cover techniques described in the next section.

\section{MINIMAL DYNAMIC COVER TECHNIQUES}

Let $N_{l}(\lambda)$ be the $(p-r) \times p$ left proper nullspace basis of $G(\lambda)$ constructed in (6). In this section we will address the following computational problem encountered when computing simple proper bases or when computing linear combination of basis vectors with least McMillan degree: given a row vector $h$, determine the output injection matrix $K$ such that the vector $v(\lambda):=h \widetilde{N}_{l}(\lambda)$ has least McMillan degree, where $\widetilde{N}_{l}(\lambda)$ is given by

$$
\widetilde{N}_{l}(\lambda)=C_{l}\left(\lambda E_{l}-A_{l}-K C_{l}\right)^{-1}\left(B_{l}+K D_{l}\right)+D_{l}
$$

As already mentioned, minimal dynamic cover techniques can be employed to perform this computation.
Computational procedures of minimal dynamic covers are presented in Varga (2004). The general idea of the cover algorithms is to perform a preliminary orthogonal similarity transformation on the system matrices in (6) by applying a special version of the controllability staircase form algorithm (see for example Varga (1990a)) to the descriptor pair $\left(A_{l}^{T}-\lambda E_{l}^{T},\left[C_{l}^{T} h^{T} C_{l}^{T}\right]\right)$ and then with additional block permutations and non-orthogonal block row/column transformations, the transformed system matrices are put in a special form which allows to cancel the maximum number of poles. For the so-called Type I dynamic covers, two nonsingular transformation matrices $U$ and $V$ result such that

$$
\begin{gathered}
U\left(A_{l}-\lambda E_{l}\right) V=\left[\begin{array}{c|c}
\widehat{A}_{11}-\lambda E_{11} & \widehat{A}_{12}-\lambda E_{12} \\
\hline \widehat{A}_{21} & \widehat{A}_{22}-\lambda E_{22}
\end{array}\right], \\
U B_{l}=\left[\frac{\widehat{B}_{1}}{\widehat{B}_{2}}\right], \quad\left[\frac{C_{l}}{h C_{l}}\right] V=\left[\begin{array}{c|c}
\widehat{C}_{11} & \widehat{C}_{12} \\
\hline 0 & \widehat{c}_{22}
\end{array}\right],
\end{gathered}
$$

where the pairs $\left(\widehat{A}_{11}-\lambda E_{11}, \widehat{C}_{11}\right)$ and $\left(\widehat{A}_{22}-\lambda E_{22}, \widehat{c}_{22}\right)$ are observable, and the submatrices $\widehat{C}_{11}$ and $\widehat{A}_{21}$ have the particular structure

$$
\left[\begin{array}{l}
\widehat{A}_{21} \\
\widehat{C}_{11}
\end{array}\right]=\left[\begin{array}{ll}
0 & A_{21} \\
0 & C_{11}
\end{array}\right]
$$

with $C_{11}$ having full column rank. By taking

$$
K=V\left[\begin{array}{c}
0 \\
K_{2}
\end{array}\right]
$$

with $K_{2}$ satisfying $K_{2} C_{11}+A_{21}=0$, we annihilate $\widehat{A}_{21}$, and thus make all eigenvalues of $\widehat{A}_{11}-\lambda E_{11}$ unobservable. The resulting vector $v(\lambda)$ of least McMillan degree, obtained by deleting the unobservable part, has the minimal state space realization

$$
v(\lambda)=\left[\begin{array}{c|c}
\widehat{A}_{22}+K_{2} \widehat{C}_{12}-\lambda E_{22} & \widehat{B}_{2}+K_{2} D_{l} \\
\hline \widehat{c}_{22} & h D_{l}
\end{array}\right]
$$

This is the typical form of achieved realizations for the basis vectors (8) of a simple basis or of a linear combination of vectors in a simple basis (9).

\section{OPERATIONS INVOLVING NULLSPACE BASES}

When solving fault detection problems, besides the computation of the nullspace basis, operations with the basis matrix (e.g., left multiplications, left coprime factorization) are necessary. For example, the following computation is necessary to check the existence conditions of the solution of the fault detection problem: Given the $p \times m$ rational matrix $G(\lambda)$ and the $p \times q$ rational matrix $F(\lambda)$, with the joint state space realization

$$
[G(\lambda) F(\lambda)]=\left[\begin{array}{c|cc}
A-\lambda E & B & B_{f} \\
\hline C & D & D_{f}
\end{array}\right],
$$

and the left minimal proper nullspace basis $N_{l}(\lambda)$ of $G(\lambda)$ in (6), compute a state space realization of $N_{l}(\lambda) F(\lambda)$. Also multiplications with $\widetilde{N}_{l}(\lambda)$ in $(10)$ and $v(\lambda)$ in $(9)$ are important when employing recursive techniques to design fault detection and isolation filters Varga (2007a,b). 
The determination of a state space realization of $N_{l}(\lambda) F(\lambda)$ can be done by computing a minimal realization of the state space realization of this rational matrix product. The numerical difficulties related to threshold based rank decisions in staircase forms based computations (Varga, 1990a) can be completely avoided by deriving explicit state space realization for this product. This can be obtained as a natural byproduct of the nullspace computation procedure.

Let $Q$ be the orthogonal transformation matrix used in computing the Kronecker-like form (4). Then, we obtain

$$
Q\left[\begin{array}{c}
B_{f} \\
D_{f}
\end{array}\right]=\left[\begin{array}{c}
* \\
\hline \widetilde{B}_{f} \\
\widetilde{D}_{f}
\end{array}\right],
$$

where the row partitioning of the right hand side corresponds to the column partitioning of $\widetilde{Y}_{l}(\lambda)$ in $(5)$. It easy to show now that

$$
N_{l}(\lambda) F(\lambda)=\widetilde{Y}_{l}(\lambda) Q\left[\begin{array}{c}
B_{f} \\
D_{f}
\end{array}\right]=C_{l}\left(\lambda E_{l}-A_{l}\right)^{-1} \widetilde{B}_{f}+\widetilde{D}_{f}(
$$

In a similar way we can compute $\tilde{N}_{l}(\lambda) F(\lambda)$. The corresponding realization is

$$
\widetilde{N}_{l}(\lambda) F(\lambda)=C_{l}\left(\lambda E_{l}-A_{l}-K C_{l}\right)^{-1}\left(\widetilde{B}_{f}+K \widetilde{D}_{f}\right)+\widetilde{D}_{f}
$$

Finally, to compute $v(\lambda) F(\lambda)$, where $v(\lambda)$ is given in (9), we observe that $v(\lambda)=h \widetilde{N}_{l}(\lambda)$, and thus

$$
v(\lambda) F(\lambda)=h C_{l}\left(\lambda E_{l}-A_{l}-K C_{l}\right)^{-1}\left(\widetilde{B}_{f}+K \widetilde{D}_{f}\right)+h \widetilde{D}_{f}
$$

Since $K$ has been obtained from the cover algorithm, the minimal realization of $v(\lambda)$ after eliminating the unobservable part is given in (11). The corresponding realization of $v(\lambda) F(\lambda)$ is

$$
v(\lambda) F(\lambda)=\left[\begin{array}{c|c}
\widehat{A}_{22}+K_{2} \widehat{C}_{12}-\lambda E_{22} & \widehat{B}_{f, 2}+K_{2} \widetilde{D}_{f} \\
\hline \widehat{c}_{22} & h \widetilde{D}_{f}
\end{array}\right]
$$

where

$$
U \widetilde{B}_{f}=\left[\begin{array}{l}
\widehat{B}_{f, 1} \\
\widehat{B}_{f, 2}
\end{array}\right]
$$

\section{APPLICATIONS TO FAULT DETECTION}

We consider linear time-invariant systems described by input-output relations of the form

$$
\mathbf{y}(\lambda)=G_{u}(\lambda) \mathbf{u}(\lambda)+G_{d}(\lambda) \mathbf{d}(\lambda)+G_{f}(\lambda) \mathbf{f}(\lambda),
$$

where $\mathbf{y}(\lambda), \mathbf{u}(\lambda), \mathbf{d}(\lambda)$, and $\mathbf{f}(\lambda)$ are Laplace- or Ztransformed vectors of the $p$-dimensional system output vector $y(t), m_{u}$-dimensional control input vector $u(t), m_{d^{-}}$ dimensional disturbance vector $d(t)$, and $m_{f}$-dimensional fault signal vector $f(t)$, respectively, and where $G_{u}(\lambda)$, $G_{d}(\lambda)$ and $G_{f}(\lambda)$ are the TFMs from the control inputs to outputs, disturbances to outputs, and fault signals to outputs, respectively.

Fault Detection Problem (FDP): Determine a proper and stable linear residual generator having the general form

$$
\mathbf{r}(\lambda)=R(\lambda)\left[\begin{array}{l}
\mathbf{y}(\lambda) \\
\mathbf{u}(\lambda)
\end{array}\right]
$$

such that: $(i) r(t)=0$ when $f(t)=0$ for all $u(t)$ and $d(t)$; and $(i i) r(t) \neq 0$ when $f_{i}(t) \neq 0$, for $i=1, \ldots, m_{f}$. Besides the above requirements it is often required for practical use that the TFM of the detector $R(\lambda)$ has the least possible McMillan degree. Note that as fault detector, we can always choose $R(\lambda)$ as a rational row vector.

The requirements $(i)$ and $(i i)$ can be easily transcribed in equivalent algebraic conditions. The (decoupling) condition $(i)$ is equivalent to

$$
R(\lambda) G(\lambda)=0
$$

where

$$
G(\lambda)=\left[\begin{array}{cc}
G_{u}(\lambda) & G_{d}(\lambda) \\
I_{m_{u}} & 0
\end{array}\right]
$$

while the (detectability) condition $(i i)$ is equivalent to

$$
R_{f_{i}}(\lambda) \neq 0, \quad i=1, \ldots, m_{f}
$$

where $R_{f_{i}}(\lambda)$ is the $i$-th column of

$$
R_{f}(\lambda):=R(\lambda)\left[\begin{array}{c}
G_{f}(\lambda) \\
0
\end{array}\right]
$$

Let $G_{f_{i}}(\lambda)$ be the $i$-th column of $G_{f}(\lambda)$. A necessary and sufficient condition for the existence of a solution is the following one (Ding and Frank, 1991; Nyberg, 2002):

Theorem 5. For the system (14) the FDP is solvable if and only if

$$
\operatorname{rank}\left[G_{d}(\lambda) G_{f_{i}}(\lambda)\right]>\operatorname{rank} G_{d}(\lambda), \quad i=1, \ldots, m_{f}
$$

From (16) it appears that $R(\lambda)$ is a left annihilator of $G(\lambda)$, thus one possibility to determine $R(\lambda)$ is to compute first a left minimal basis $N_{l}(\lambda)$ for the left nullspace of $G(\lambda)$, and then to build a stable scalar output detector as

$$
R(\lambda)=h(\lambda) N_{l}(\lambda)
$$

representing a linear combination of the rows of $N_{l}(\lambda)$, such that conditions (18) are fulfilled. The above expression represents a parametrization of all possible detectors and is the basis of the nullspace methods.

Consider a descriptor state space realization of (14)

$$
\begin{aligned}
E \lambda x(t) & =A x(t)+B_{u} u(t)+B_{d} d(t)+B_{f} f(t) \\
y(t) & =C x(t)+D_{u} u(t)+D_{d} d(t)+D_{f} f(t)
\end{aligned}
$$

where $\lambda x(t)=\dot{x}(t)$ or $\lambda x(t)=x(t+1)$ depending on the type of the system, continuous or discrete, respectively. Using the methods described in Section 3, we compute first a minimal proper left nullspace basis $N_{l}(\lambda)$ of $G(\lambda)$ defined in (17). The state space realization of the $(p-r) \times\left(p+m_{u}\right)$ TFM $N_{l}(\lambda)$ is given by (6), where $r$ is the rank of $G_{d}(\lambda)$.

To check the existence conditions of Theorem 5, we use (13) to compute

$N_{f}(\lambda):=N_{l}(\lambda)\left[\begin{array}{c}G_{f}(\lambda) \\ 0\end{array}\right]=C_{l}\left(\lambda E_{l}-A_{l}\right)^{-1} \widetilde{B}_{f}+\widetilde{D}_{f}$

where $Q\left[\begin{array}{lll}B_{f}^{T} & D_{f}^{T} & 0\end{array}\right]^{T}=\left[*\left|\widetilde{B}_{f}^{T}\right| \widetilde{D}_{f}^{T}\right]^{T}$. Since the pair $\left(A_{l}-\lambda E_{l}, C_{l}\right)$ is observable, checking the condition (18) is equivalent to verify that

$$
\left[\begin{array}{c}
\widetilde{B}_{f_{i}} \\
\widetilde{D}_{f_{i}}
\end{array}\right] \neq 0, \quad i=1, \ldots, m_{f}
$$


where $\widetilde{B}_{f_{i}}$ and $\widetilde{D}_{f_{i}}$ denote the $i$-th columns of $\widetilde{B}_{f}$ and $\widetilde{D}_{f}$, respectively. An important application of these formulas is the determination of the complete achievable fault influence structure in connection with the fault detection and isolation problem (FDIP) (Varga, 2007a).

Similar formulas as above can be employed to check the detectability conditions (18) when determining a least order detector by building candidate detectors as linear combinations of the form $R(\lambda)=h \widetilde{N}_{l}(\lambda)$ (see (9) and (10)) using suitably chosen $h$ and $K$. More details are provided in (Varga, 2007a).

Another important application of the formulas developed in Section 6 allows the efficient solution of the FDIP, using a bank of scalar detectors with least dynamical order. A two steps procedure has been proposed recently by Varga (2007b), where in the first step a complete decoupling of control and disturbance inputs is achieved using a preliminary raw detector $N_{l}(\lambda)$. The resulting fault-toresidual influence (without control and disturbance inputs) can be expressed as

$$
\widetilde{\mathbf{y}}(\lambda):=N_{f}(\lambda) \mathbf{f}(\lambda),
$$

where a realization of $N_{f}(\lambda)$ is given by (23). The system (24) has generally a reduced McMillan degree (Varga, 2007a) and also a reduced number of outputs $p-r$, thus the solution of the FDIP by designing a bank of scalar output detectors is usually simpler than for the original problem. The second step involves the successive solution of simpler FDPs, where the formulas of Section 6 serve to check the solvability conditions and to update the detector, in a completely similar way as done in the first step. The overall design procedure avoids completely any need to explicitly compute minimal realizations of the resulting detectors and therefore is perfectly suitable for robust software implementation.

\section{CONCLUSIONS}

The rational nullspace computation based techniques to solve the FDP or FDIP allow a flexible approach to address different aspects of these problems, like computing least order detectors, checking existence conditions, computing the achievable fault influence structure, or employing updating techniques to design a bank of detectors to solve the FDIP. The underlying computations extensively use orthogonal similarity transformations to perform the important computational steps, as for example, to determine a proper nullspace basis or to check the existence conditions of a solution. In contrast, methods based on polynomial nullspace computations are less flexible, and involve computational detours, which are highly questionable from a numerical point of view.

Simple proper rational bases are the direct correspondents of polynomial basis and can be computed using the proposed numerical algorithms based on minimal cover techniques. Using such a basis, the least order FDP can be easily solved. Interestingly, this type of basis is not really necessary for this purpose, since a more efficient approach can be devised by computing linear combinations of a simple basis vectors directly from a proper minimal nullspace basis. This aspect is relevant for implementing robust numerical software as those available in a recent FAult Detection Toolbox for Matlab (Varga, 2006).

\section{REFERENCES}

E.N. Antoniou, A.I.G. Vardulakis, and S. Vologiannidis. Numerical computation of minimal polynomial bases: A generalized resultant approach. Lin. Alg. \& Appl., 405: 264-278, 2005.

Th.G.J. Beelen. New Algorithms for Computing the Kronecker structure of a Pencil with Applications to Systems and Control Theory. Ph. D. Thesis, Eindhoven University of Technology, 1987.

X. Ding and P. M. Frank. Frequency domain approach and threshold selector for robust model-based fault detection and isolation. Proc. of IFAC Symposium SAFEPROCESS 1991, Baden-Baden, Germany, 1991.

G. D. Forney. Minimal bases of rational vector spaces with applications to multivariable linear systems. SIAM J. Control, 13:493-520, 1975.

E. Frisk and M. Nyberg. A minimal polynomial basis solution to residual generation for fault diagnosis in linear systems. Automatica, 37:1417-1424, 2001.

T. Kailath. Linear Systems. Prentice Hall, Englewood Cliffs, N.J., 1980.

M. Nyberg. Criterions for detectability and strong detectability of faults in linear systems. Int. J. Control, 75:490-501, 2002.

A. I. G. Vardulakis and N. Karcanias. Proper and stable, minimal MacMillan degrees bases of rational vector spaces. IEEE Trans. Automat. Control, 29:1118 - 1120, 1984.

A. Varga. Computation of irreducible generalized statespace realizations. Kybernetika, 26:89-106, 1990.

A. Varga. Computation of Kronecker-like forms of a system pencil: Applications, algorithms and software. Proc. CACSD'96 Symposium, Dearborn, MI, pages 7782,1996

A. Varga. On computing least order fault detectors using rational nullspace bases. Proc. of IFAC Symp. SAFEPROCESS'2003, Washington D.C., 2003.

A. Varga. Reliable algorithms for computing minimal dynamic covers for descriptor systems. Proc. of $M T N S^{\prime} 04$, Leuven, Belgium, 2004.

A. Varga. A FAult Detection toolbox for Matlab. Proc. of CACSD'06, Munich, Germany, 2006.

A. Varga. Design of Least Order Residual Generators for Fault Detection and Isolation with Application to Monitoring Actuator/Surface Faults for a Boeing 747 100/200 Aircraft, GARTEUR AG-16 TP-2007-1. Technical report, DLR Oberpfaffenhofen, Inst. of Robotics and Mechatronics, May 2007a.

A. Varga. Fault detection and isolation of actuator failures for a large transport aircraft. Proc. First CEAS European Air and Space Conference, Berlin, Germany, 2007b.

A. Varga. On designing least order residual generators for fault detection and isolation. Proc. 16th Internat. Conf. on Control Systems and Computer Science, Bucharest, Romania, pages 323-330, 2007c.

M. E. Warren and A. E. Eckberg. On the dimension of controllability subspaces: A characterization via polynomial matrices and Kronecker invariants. SIAM J. Contr., 13: 434-445, 1975. 